\title{
Trucut Biopsy of a Solitary Focal Metastatic Superior Vena Cava Thrombus via Percutaneous Transjugular Approach Using Real-Time Fluoroscopic Needle Visualization in Two Planes
}

\author{
Amey Narkhede ${ }^{1} \quad$ Ajit Yadav ${ }^{1}$ Gaurav Gangwani ${ }^{1} \quad$ Ali Asgar Sabir ${ }^{1} \quad$ Arun Gupta ${ }^{1}$ \\ ${ }^{1}$ Department of Interventional Radiology, Sir Gangaram Hospital, \\ New Delhi, India \\ Address for correspondence Arun Gupta, MBBS, MD, 20A, \\ Department of Interventional Radiology, Sir Ganga Ram Hospital, \\ Rajinder Nagar, New Delhi 110060, India \\ (e-mail: irdrarungupta@gmail.com).
}

\begin{abstract}
Keywords

- intravascular biopsy

- intracaval metastasis

- superior vena cava

syndrome

Presentation of metastasis as a solitary focal lesion at an intravascular location is encountered quite infrequently. Owing to its intravascular location, accessing the lesion for sampling and obtaining an adequate amount of tissue are technically difficult. Among the various methods of obtaining an adequate sample from intravascular lesions, scoop and trucut biopsies appear to be safer and more advantageous. The authors present a case of a 65 -year-old woman with symptoms of superior vena cava syndrome secondary to a solitary focal fluorodeoxyglucose (FDG)-avid thrombus within the superior vena cava lumen 14 years after complete remission of breast carcinoma. This lesion was approached via transjugular venous access and biopsied under fluoroscopic guidance with continuous contrast injection and real-time needle visualization in two planes.
\end{abstract}

\section{Introduction}

Presentation of metastasis in the form of a solitary focal intravascular thrombus is an infrequent occurrence. Sampling an intravascular lesion for histopathologic examination presents as a challenging task because it is important to obtain an adequate sample while minimizing the complications related to this procedure. Differentiation of a malignant intravascular thrombus from a bland thrombus is an important step in the management, especially in a known case of carcinoma, because frequency of both types of thrombi is known to increase in malignancy. Although imaging helps in differentiation between these two, biopsy remains the gold standard for diagnosis as well as initiating treatment.

\section{Case Report}

A 65-year-old woman presented with a chief complaint of chest tightness for 2 months. She had history of rightsided modified radical mastectomy 14 years prior to the presentation and had received adjuvant chemotherapy and radiotherapy. The postoperative specimen biopsy report was suggestive of grade II infiltrating ductal carcinoma. Estrogen receptor/progesterone receptor (ER/PR) was $100 \%$ positive, with metastatic ipsilateral axillary lymph nodes. The patient continued hormonal therapy for 10 years postsurgery.

Chest radiograph revealed moderate left pleural effusion with fibroparenchymal changes in the left mid zone. Pleural fluid analysis was negative for malignant cells. Noncontrast multidetector computed tomography (CT) of the chest revealed moderate left pleural effusion, irregular area of consolidation in left upper lobe, and subtle ground-glass haze with fibroatelectatic changes in the bilateral lung fields. Stranding was noted in the prevascular and retrosternal regions. Positron emission tomography-computed tomography (PET-CT) was suggestive of fluorodeoxyglucose (FDG)-avid focal thrombus within the lumen of the superior vena cava (SVC) causing approximately $90 \%$ luminal stenosis and resulting in formation of venovenous collaterals ( - Fig. 1). received

May 2, 2018

accepted after revision

June 21, 2018

published online

August 17, 2018
DOI https://doi.org/

$10.1055 / \mathrm{s}-0038-1668176$.

ISSN 2457-0214.
Copyright $\odot 2018$ by Indian Society of Vascular and Interventional Radiology
License terms

(ㅇ) (1) $\ominus \circledast$ 


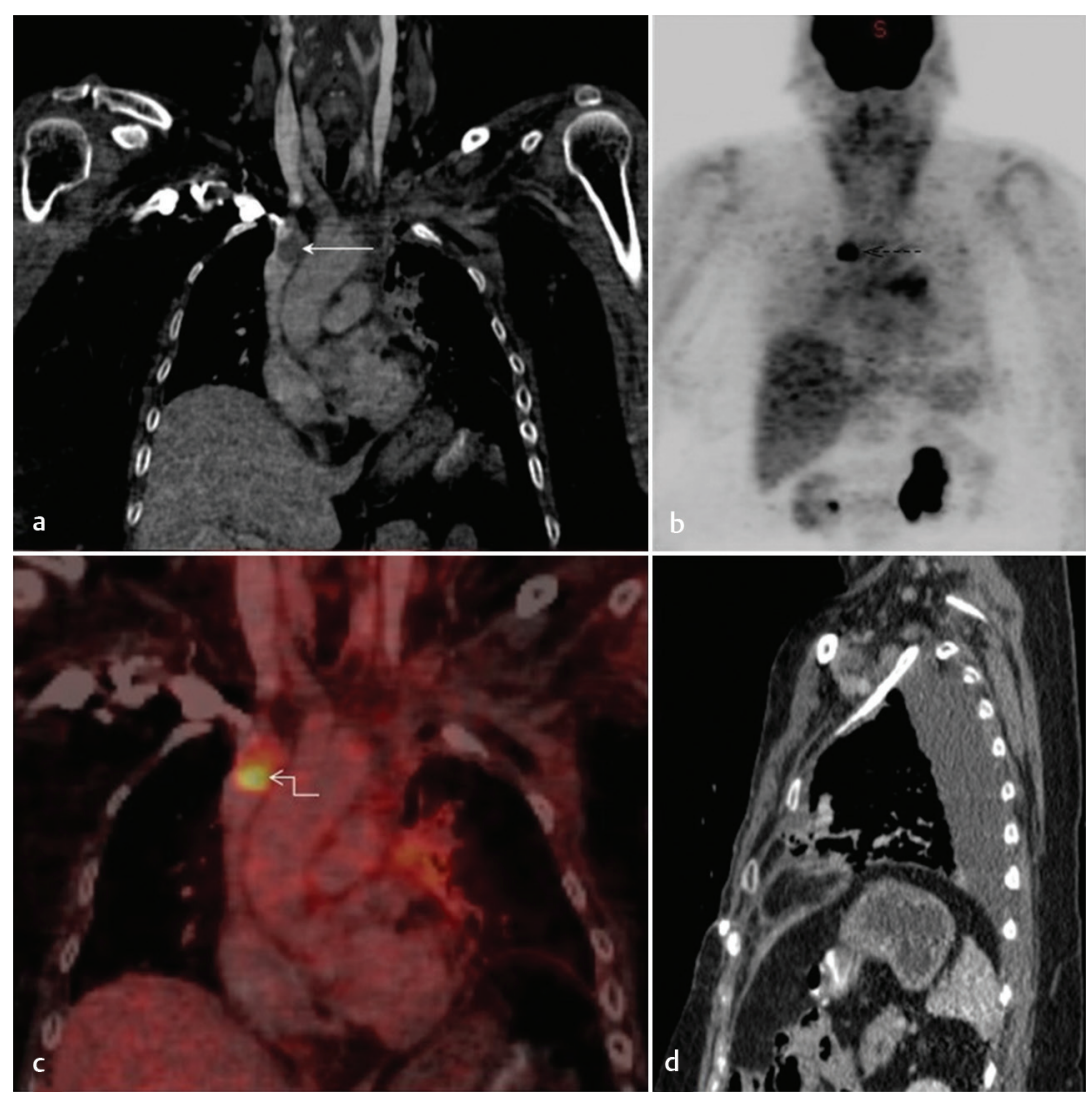

Fig. 1 Positron emission tomography-computed tomography (PET-CT). (a) Coronal reformatted CT image reveals focal thrombus (straight arrow) in the proximal superior vena cava (SVC) lumen attached to its medial wall. (b) PET image (coronal AP projection) reveals focal FDG-avid area (dashed arrow) in the corresponding location of the thrombus. (c) Fused PET-CT coronal image suggestive of FDG-avid intraluminal SVC thrombus (square arrow). (d) Sagittal reformatted CT image of the thorax through left midaxillary line is suggestive of moderate pleural effusion.

No other FDG-avid areas were noted on the PET scan. NonFDG-avid thrombi were noted in the left brachiocephalic and subclavian veins. The patient's symptoms progressed during the course with development of SVC syndrome (difficulty breathing, headache, eye engorgement, venous distention in the neck, and distended veins in the upper chest). Sampling of the thrombus was deemed necessary to confirm malignant etiology so that management could be initiated.

Under all aseptic precautions, local anesthesia, and sonographic guidance, the right internal jugular vein was accessed, and a $6 \mathrm{~F}$ vascular sheath was placed. A 0.035-in hydrophilic guidewire (Terumo) and 5F Kumpe catheter (Cook Medical) was advanced up to the cavoatrial junction. Venography revealed a focal filling defect in the proximal SVC, predominantly along its medial margin, corresponding to the metabolically active thrombus on PET-CT ( $\mathbf{- F i g . ~ 2 a ) . ~}$ The anterior location of the thrombus was confirmed repeating the venogram in lateral projection $(-\mathbf{F i g}$. 2b). The sheath was advanced to just few inches short of the thrombus. A 14G metal cannula $(53.5 \mathrm{~cm})$ was used from the LABS-100 biopsy set (Cook Medical) and positioned in the sheath to the proximal margin of the filling defect. The distal-end angulation of the metal cannula was reduced manually ( 15 degrees) to obtain a straight path. The quick-core biopsy needle from the set $(18 \mathrm{G}-60 \mathrm{~cm})$ was inserted within the metal cannula and under continuous contrast injection via the sheath. The final throw end $(20 \mathrm{~mm})$ was pushed into the filling defect under real-time fluoroscopic visualization in both anteroposterior and lateral projections. Tissue core from the thrombus was obtained ( $\mathbf{- F i g}$. $2 \mathbf{c})$. The entire procedure was repeated to acquire another core under similar guidance. Postprocedure venogram revealed focal ulceration within the thrombus ( - Fig. 2d).

The histopathology report was suggestive of metastatic breast carcinoma (possibly lobular carcinoma). Tumor cells were positive for ER ( 90\% strong), PR (> 60\%) and P63 (occasional cells only). Her2neu was negative (1+). Tumor cells were negative for thyroid transcription factor 1 .

The signs of SVC syndrome progressed significantly in the next 2 days. The right internal jugular vein (IJV) showed presence of a postprocedure bland thrombus commencing at the previous transjugular puncture site. A transfemoral venous access was obtained. Aspiration thrombectomy followed by a bolus infusion of $3 \mathrm{mg}$ of t-PA (tissue-plasminogen activator) was performed. No change in the extent or size of the bland thrombus was seen. A $14 \times 40-\mathrm{mm}$ uncovered vascular stent (Bard-E-luminex) was placed across the malignant tumor thrombus ( - Fig. $\mathbf{3 a}, \mathbf{b}$ ). The prestenting gradient of $8 \mathrm{~mm} \mathrm{Hg}$ (12-4) was reduced to $2 \mathrm{~mm} \mathrm{Hg}(10-8)$ after stenting. Enoxaparin was resumed post stenting.

The signs of SVC syndrome immediately showed improvement. However, the patient developed dyspnea 6 hours post procedure managed by dual bilevel positive airways pressure 
(BiPAP) settings. Palliative chemotherapy (Paclitaxel) was initiated. Radiotherapy was withheld until the clinical condition further improved. The dyspnea improved gradually,

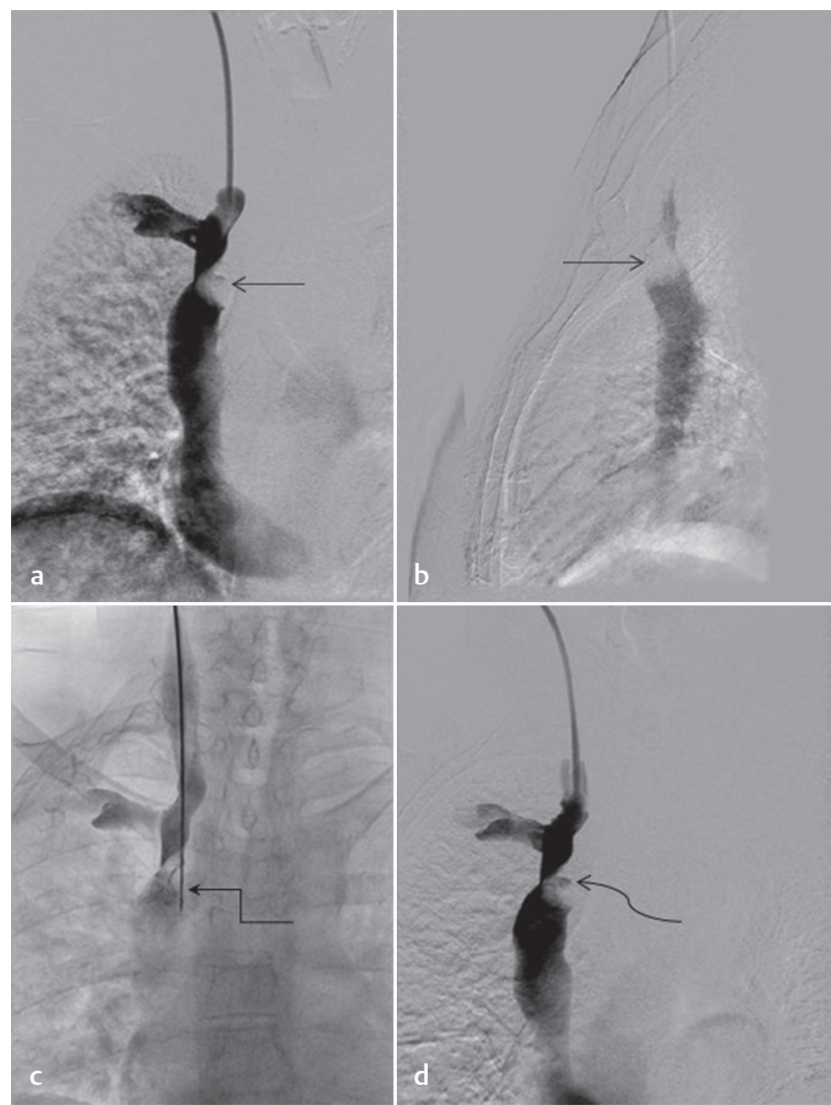

Fig. 2 Digital subtraction angiography images with right jugular access: (a) PA and (b) lateral projections during venography reveal a focal filling defect (straight arrows) in the superior vena cava (SVC) lumen along its medial and anterior wall. (c) Biopsy of the thrombus being done under real-time fluoroscopy guidance using cook biopsy needle (squared arrow) with continuous contrast injection via the jugular sheath. (d) Postbiopsy right jugular venogram reveals focal ulceration (curved arrow) in the central aspect of the intraluminal SVC filling defect. and the patient was showing improvement, but sudden unexpected death occurred after 3 days. Cause of death was unknown, and the relatives decided not to allow autopsy. Pulmonary thromboembolism was hypothesized to be the probable cause of death.

\section{Discussion}

Intravascular metastases are known to occur more frequently in hepatocellular, renal, lung, and gonadal carcinomas as compared with other carcinomas. The incidence of intravascular metastasis is a relatively less described phenomenon in breast carcinoma as it commonly presents with bland thrombi involving the extremities during the first 6 months after diagnosis. Additionally it is quite rare to find a solitary focal vena caval metastatic thrombus presenting as a recurrence after complete remission treated by modified radical mastectomy, adjuvant chemotherapy, radiotherapy, and 10 years of hormonal therapy. If found, the metastatic thrombus mostly occurs in the inferior vena cava (IVC). ${ }^{1}$ Occurrence of SVC metastasis is rare with only few case reports published. ${ }^{2}$

The definitive method to establish the malignant etiology of a lesion is through histopathology. To obtain a biopsy sample from intracaval region, a proper balance has to be established between the risk factors associated with each option available and the probable success rate of obtaining an adequate sample.

Various methods to obtain a biopsy sample from SVC mass include open thoracotomy, percutaneous image guided biopsy, and transluminal endovascular biopsy. Minimally invasive intravascular approach, that is, transjugular intracaval biopsy in our case, is favored because it provides multiple advantages over percutaneous or open chest sampling of the mediastinal tissue. This can be done in critical patients and patients with multiple comorbidities. The life-threatening complications such as pneumothorax, vascular injury, and air embolism are avoided, which can occur during percutaneous biopsy. The risk of dissemination is also reduced because the

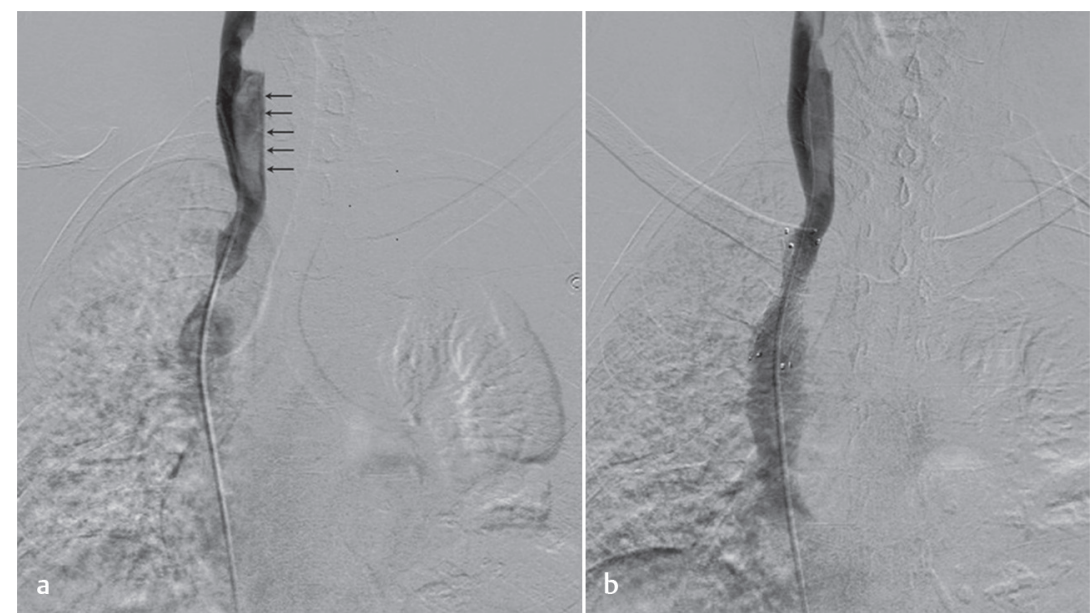

Fig. 3 Superior vena cava (SVC) stenting: (a) Right internal jugular venogram reveals the focal filling defect in the SVC lumen with resultant high-grade luminal stenosis. Note is made of longitudinal filling defect (straight arrows) in the right internal jugular vein lumen likely s/o acute thrombus as the result of the previous jugular access for biopsy. (b) Right internal jugular venography post stent deployment in the SVC lumen reveals normal antegrade flow in the SVC with minimal residual stenosis. 
vascular wall is not traversed by intravascular core biopsy needle as in percutaneous biopsy.

The procedure for performing percutaneous transluminal intracaval biopsy using "road-map" technique was first described by Jackson and Adam in 1991. ${ }^{3}$ In our case instead of using the road map for guidance, we targeted the region of persistent filling defect under fluoroscopic guidance using continuous contrast injection. We believe this technique to be more precise because it allows real-time visualization of the filling defect, hence increasing the probability of obtaining successful biopsy. However, Kim et al have presented a different technique for percutaneous intracaval biopsy of an intraluminal mass within the IVC, using sonographic guidance. They have proposed that it is advantageous to use sonography over conventional fluoroscopy because it can visualize directly the contact of the biopsy forceps with the target lesion. ${ }^{4}$ However, this technique is not feasible in the SVC in all patients, because of poor sonographic window.

Various methods are used to obtain tissue from an intracaval lesion. These include transcatheter aspiration, ${ }^{5}$ brush biopsy, ${ }^{6}$ scoop biopsy, ${ }^{7}$ endoscopic biopsy forceps, Simpson atherectomy catheter, ${ }^{8}$ endomyocardial bioptome, and trucut biopsy using the transjugular liver biopsy set. ${ }^{9}$

The transcatheter aspiration and brush biopsy are techniques of the past. They provide a small sample suitable only for cytology. The transcatheter aspiration requires that the tumor thrombus should be friable. ${ }^{3}$ As compared with these two, all the other methods provide larger tissue samples. Endoscopic biopsy forceps and endomyocardial bioptome pose a threat of perforation because there is biting and pulling of the tissue. Scoop biopsy forceps and trucut biopsy are relatively safer due to controlled slicing and retrieval of tissue with minimum damage to the targeted tissue. ${ }^{7}$ An inherent disadvantage of each technique is the possibility of disseminating the metastatic tissue as emboli into the pulmonary circulation.

In our case, we took fluoroscopic and angiographic guidance to approach the tumor thrombus in the SVC. The focal filling defect was in the proximal SVC on the venogram that was confirmed to be anteromedial in location on anteroposterior and lateral views and was targeted. The biopsy set we used in our case was semiautomatic, which provided us with better maneuvering of the needle during biopsy. We pointed the throw direction away from the vessel wall in two planes, avoiding any chances of perforation.

Presence of an intraluminal SVC thrombus can lead to SVC syndrome. The treatment options for this have been radiotherapy or chemotherapy or both. Use of stents has mostly been a supportive tool for palliation. However, a few authors have suggested using endovascular stents as the first line of treatment because there is immediate relief from the symptoms of obstruction as we observed in our case. ${ }^{10}$

In conclusion, solitary metastatic thrombus occurring in the SVC presenting with symptoms of SVC syndrome, years after complete treatment of a breast carcinoma, is a rarity. Obtaining a biopsy from such a critical location to prove its metastatic etiology stands as a challenging task. We conclude that obtaining a trucut core biopsy via a transjugular intracaval approach using continuous contrast injection under fluoroscopic guidance in two planes is safe and effective to obtain adequate sample from a SVC lesion.

\section{Disclosures}

None.

\section{Acknowledgments}

None.

\section{References}

1 Lai P, Bomanji JB, Mahmood S, et al. Detection of tumour thrombus by $18 \mathrm{~F}-\mathrm{FDG}-\mathrm{PET} / \mathrm{CT}$ imaging. Eur J Cancer Prev 2007;16(1):90-94

2 Batra S, Doval DC, Batra U, Suresh P, Dhiman A, Talwar V. Gallbladder cancer with tumor thrombus in the superior vena cava. Hepatobiliary Pancreat Dis Int 2010;9(3):325-328

3 Jackson JE, Adam A. Percutaneous transcaval tumour biopsy using a 'road-map' technique. Clin Radiol 1991;44(3):195-196

4 Kim SE, Norfray J, Heiser WJ, Foran J, Roychowdhury S. Sonographically guided intravascular biopsy of an intraluminal mass in the inferior vena cava. J Ultrasound Med 1994;13(5):405-407

5 Wendth AJ Jr, Garlick WB, Pantoja GE, Shamoun J. Transcatheter biopsy of renal carcinoma invading the inferior vena cava. J Urol 1976;115(3):331-332

6 Mills SR, Doppman JL, Head GL, Javadpour N, Brennan MF, Chu EW. Transcatheter brush biopsy of intravenous tumor thrombi. Radiology 1978;127(3):667-670

7 Kishi K, Sonomura T, Terada M, Sato M. Scoop biopsy of intracaval tumor thrombi: a preliminary report of a minimally invasive technique to obtain large samples. Eur J Radiol 1997;24(3):263-268

8 Castaneda F, Moradian G, Hunter D, Castaneda-Zuniga W, Amplatz K. Percutaneous intravascular biopsy using a Simpson atherectomy catheter: technical note. Cardiovasc Intervent Radiol 1989;12(6):342-343

9 Weinberg L, Abu-Ssaydeh D, Macgregor C, et al. Transjugular biopsy case report of inferior vena cava hepatocellular carcinoma with intracardiac extension. Int J Surg Case Rep 2017;39:324-327

10 Lanciego C, Pangua C, Chacón JI, et al. Endovascular stenting as the first step in the overall management of malignant superior vena cava syndrome. AJR Am J Roentgenol 2009;193(2):549-558 